\title{
Is the Angular Momentum of an Electron Conserved in a Uniform Magnetic Field?
}

\author{
Colin R. Greenshields, Robert L. Stamps, Sonja Franke-Arnold, and Stephen M. Barnett \\ SUPA School of Physics and Astronomy, University of Glasgow, Glasgow G12 8QQ, United Kingdom
} (Received 25 July 2014; published 9 December 2014)

\begin{abstract}
We show that an electron moving in a uniform magnetic field possesses a time-varying "diamagnetic" angular momentum. Surprisingly this means that the kinetic angular momentum of the electron may vary with time, despite the rotational symmetry of the system. This apparent violation of angular momentum conservation is resolved by including the angular momentum of the surrounding fields.

DOI: 10.1103/PhysRevLett.113.240404

PACS numbers: 03.65.-w, 11.30.-j, 41.75.-i, 42.50.Tx
\end{abstract}

Introduction.-There is an intimate relation between angular momentum and rotational symmetry, as encapsulated by Noether's theorem [1]. In particular, if a system is symmetric under rotation about a given axis, the angular momentum along that axis will be conserved. This is the case with free electron vortices, which in the last few years were predicted and observed in electron microscopes [2-5]. Electron vortex beams in field-free space have a cylindrically symmetric wave function and maintain a constant orbital angular momentum in the direction of propagation [2].

Based on the same argument of rotational symmetry it would seem that for an electron exposed to a uniform magnetic field its orbital angular momentum in the direction of the field must be conserved. This is indeed true of the angular momentum about the axis of the classical cyclotron orbit, and, furthermore, the energy eigenstates of an electron in a uniform magnetic field - the Landau states-have constant angular momentum [6,7].

On the other hand, it is known that the kinetic angular momentum of an electron, which describes its mechanical motion, is not necessarily constant even when the electron interacts with external fields which are rotationally symmetric [8]. The balance and redistribution of momentum and angular momentum between matter and fields is a fundamental problem of great general interest [9-12].

Recent investigations of vortex electron states in uniform and quasiuniform magnetic fields have revealed that the angular velocity of the electron depends not only on the field strength but also on the azimuthal quantum number and the radial position $[7,13,14]$. Furthermore, in these quantum states the average radial position of the electron is not in general constant, but rather changes as the wave function diffracts [14-16]. This contrasts sharply with the classical orbit in a uniform magnetic field, leading to the

Published by the American Physical Society under the terms of the Creative Commons Attribution 3.0 License. Further distribution of this work must maintain attribution to the author(s) and the published article's title, journal citation, and DOI. question of whether the angular momentum of the electron is in fact conserved.

In this Letter, we show that for an electron in a nonstationary state, the changing radius of the electron's probability distribution in fact gives rise to a time-varying kinetic angular momentum. The canonical angular momentum however remains constant. The apparent violation of angular momentum conservation is resolved by considering the angular momentum in the fields surrounding the electron. We show that the total kinetic angular momentum, including that of the fields, is conserved, as indeed it must be

Results.-We consider an electron moving in a uniform magnetic field, and take the direction of this field to define the $z$ axis. The (nonrelativistic) Hamiltonian for this system can be written in the form

$$
H=\frac{1}{2 m}\left(\mathbf{p}^{\mathrm{kin}}\right)^{2},
$$

where $\mathbf{p}^{\text {kin }}=m \mathbf{v}=\mathbf{p}^{\text {can }}-e \mathbf{A}$ is the kinetic momentum, $\mathbf{p}^{\mathrm{can}}=-i \hbar \nabla$ is the canonical momentum, $e=-|e|$ is the charge of the electron, and $m$ its mass. We choose a vector potential which in cylindrical polar coordinates $(\rho, \phi, z)$ has the form

$$
\mathbf{A}=\frac{B \rho}{2} \hat{\boldsymbol{\phi}} \Rightarrow \mathbf{B}=\nabla \times \mathbf{A}=B \hat{\mathbf{z}} .
$$

We note, for later reference, that this choice of vector potential corresponds to the Coulomb gauge, in that $\nabla \cdot \mathbf{A}=0$. The Hamiltonian (1) can then be rewritten as [17]

$$
H=\frac{1}{2 m}\left(\mathbf{p}^{\mathrm{can}}\right)^{2}+\frac{1}{2} m \omega_{L}^{2} \rho^{2}+\omega_{L} L_{z}^{\mathrm{can}},
$$

where $\omega_{L}=-e B /(2 m)$ is the Larmor frequency and

$$
L_{z}^{\mathrm{can}}=\left(\mathbf{r} \times \mathbf{p}^{\mathrm{can}}\right)_{z}=-i \hbar \frac{\partial}{\partial \phi}
$$

is the $z$ component of canonical orbital angular momentum. As the $z$ component of linear momentum $p_{z}^{\text {kin }}=p_{z}^{\text {can }}$ commutes with the Hamiltonian, the motion of the electron 
in the $z$ direction is unaffected by the magnetic field. This means that for an electron beam propagating along the $z$ axis we need only consider the $z$ component of angular momentum. Furthermore, as the Hamiltonian, in our chosen gauge, is independent of $\phi$ it commutes with the canonical angular momentum $\left[L_{z}^{\text {can }}, H\right]=0$ and, hence, $L_{z}^{\text {can }}$ is conserved [15].

Consider an electron wave function with cylindrical symmetry so that

$$
\Psi=u(\rho, z, t) e^{i \ell \phi} .
$$

We make no assumption about the form of the function $u$, so that in general this will not be an energy eigenstate. This state is, however, an eigenstate of $L_{z}^{\text {can }}$ and hence the expectation value of its angular momentum has the timeindependent value

$$
\left\langle L_{z}^{\text {can }}\right\rangle=\ell \hbar .
$$

This is eminently reasonable as the system is symmetric under rotation about the $z$ axis, so that according to Noether's theorem the $z$ component of the angular momentum should be conserved.

In the presence of a magnetic field, the kinetic orbital angular momentum differs from its canonical counterpart:

$$
L_{z}^{\mathrm{kin}}=\left(\mathbf{r} \times \mathbf{p}^{\mathrm{kin}}\right)_{z}=L_{z}^{\mathrm{can}}+m \omega_{L} \rho^{2}
$$

[8], where we have used the definition of the kinetic linear momentum and the specific form of the vector potential (2). We see that the field-dependent contribution to the kinetic angular momentum is associated with a rotation of the electron probability distribution at constant angular velocity $\omega_{L}$. This is consistent with Larmor's theorem [18] and can be interpreted as a diamagnetic response of the electron to the external magnetic field [19].

The expectation value of the kinetic orbital angular momentum can be expressed as

$$
\left\langle L_{z}^{\mathrm{kin}}\right\rangle=\ell \hbar+\left\langle I_{z}\right\rangle \omega_{L},
$$

where we have used the fact that the expectation value of the $z$ component of the electron's moment of inertia is

$$
\left\langle I_{z}\right\rangle=m\left\langle\rho^{2}\right\rangle .
$$

This means that the kinetic angular momentum of the electron will be constant only if the radial probability distribution is constant. The squared radius $\rho^{2}$ does not commute with the Hamiltonian, however, meaning this quantity is not a constant of motion [6]. This means that, in contrast with the classical motion, the mean value $\left\langle\rho^{2}\right\rangle$ will not, in general, be a constant.

It can be seen from the form of the Hamiltonian (3) that the radial coordinate exhibits a harmonic motion. This can be understood as the radial diffraction of the electron wave function in a harmonic potential generated by the interaction with the magnetic field [15]. The energy associated with the motion perpendicular to the magnetic field remains constant and has the expectation value

$$
E_{\perp}=\left\langle H-\frac{1}{2 m}\left(p_{z}^{\mathrm{kin}}\right)^{2}\right\rangle .
$$

We will obtain the time behavior of the radial width, and later also of the kinetic orbital angular momentum, from Heisenberg's formalism:

$$
\begin{aligned}
\frac{\partial^{2}\left\langle\rho^{2}\right\rangle(t)}{\partial t^{2}} & =-\frac{1}{\hbar^{2}}\left\langle\left[\left[\rho^{2}, H\right], H\right]\right\rangle(t) \\
& =-\omega_{c}^{2}\left(\left\langle\rho^{2}\right\rangle(t)-\tilde{\rho^{2}}\right),
\end{aligned}
$$

where $\omega_{c}=2 \omega_{L}=-e B / m$ is the classical cyclotron frequency and $\tilde{\rho}^{2}=\left(E_{\perp}-\omega_{L} \ell \hbar\right) /\left(m \omega_{L}^{2}\right)$ is the constant steady-state value which depends on the energy, the canonical angular momentum, and the magnetic field. It can be seen from (11) that the mean-square radius oscillates sinusoidally about the value $\tilde{\rho^{2}}$ at the cyclotron frequency. Setting $t=0$ to correspond to a stationary point of this oscillation, we have

$$
\left\langle\rho^{2}\right\rangle(t)=\tilde{\rho^{2}}+\left(\left\langle\rho^{2}\right\rangle(0)-\tilde{\rho^{2}}\right) \cos \left(\omega_{c} t\right) .
$$

According to the relation (8) this is intrinsically linked to an oscillation of the kinetic angular momentum:

$$
\left\langle L_{z}^{\mathrm{kin}}\right\rangle(t)=\tilde{L}_{z}^{\mathrm{kin}}+\left(\left\langle L_{z}^{\mathrm{kin}}\right\rangle(0)-\tilde{L}_{z}^{\mathrm{kin}}\right) \cos \left(\omega_{c} t\right),
$$

where the steady-state value of the kinetic angular momentum

$$
\tilde{L}_{z}^{\text {kin }}=\ell \hbar+m \omega_{L} \tilde{\rho^{2}}=\frac{2}{\omega_{c}} E_{\perp}
$$

coincides with the classical value of the kinetic angular momentum for an electron with rotational kinetic energy $E_{\perp}$. In general, the angular momentum oscillates sinusoidally about the classical value, with the same frequency $\omega_{c}$ as the classical cyclotron motion. Only if the kinetic angular momentum is equal to the classical value does its expectation value remain constant. In this sense (14) defines the steady-state value $\tilde{\rho^{2}}$.

We can obtain an exact solution for (12) in the case when at $t=0$ the wave function (5) has the Laguerre-Gaussian form

$$
\begin{aligned}
\Psi_{n, \ell}(0)= & \mathrm{LG}_{n, \ell} \propto\left(\frac{\rho \sqrt{2}}{\rho_{0}}\right)^{|\ell|} \exp \left(-\frac{\rho^{2}}{\rho_{0}^{2}}\right) L_{n}^{|\ell|}\left(\frac{2 \rho^{2}}{\rho_{0}^{2}}\right) \\
& \times \exp \left[i\left(\ell \phi+k_{z} z\right)\right],
\end{aligned}
$$


where $\ell \in \mathbb{Z}, n=0,1,2, \ldots$, and $L_{n}^{|\ell|}$ is an associated Laguerre polynomial. The index $n$ specifies the number of radial nodes in the wave function, while $\rho_{0}$ is the width of the Gaussian envelope. The Laguerre-Gaussian wave functions can be used to describe electron vortex beams which have intrinsic orbital angular momentum $\ell \hbar$ [2] as well as electron beams with no intrinsic orbital angular momentum in the case when $\ell=0$.

The mean-square radius of the Laguerre-Gaussian wave function (15) is equal to

$$
\left\langle\rho^{2}\right\rangle_{n, \ell}(0)=\frac{1}{2}(2 n+|\ell|+1) \rho_{0}^{2}
$$

[20]. As this depends on the radial index $n$, it follows that the kinetic angular momentum of the electron also depends on $n$, which is not the case in the absence of a magnetic field. The steady-state mean-square radius for the same electron energy obtained from (10) and (14) is

$$
{\tilde{\rho^{2}}}_{n, \ell}=\frac{1}{4}(2 n+|\ell|+1)\left[1+\left(\frac{\rho_{B}}{\rho_{0}}\right)^{4}\right] \rho_{0}^{2},
$$

where $\rho_{B}^{2}=2 \hbar /\left(m\left|\omega_{L}\right|\right)=4 \hbar /|e B|$. As the phase of the wave function (15) does not depend on $\rho$, we have

$$
\frac{\partial\left\langle\rho^{2}\right\rangle_{n, \ell}(0)}{\partial t}=\frac{1}{\hbar} \operatorname{Im}\left\langle\left[\rho^{2}, H\right]\right\rangle_{n, \ell}(0)=0,
$$

meaning that this wave function corresponds to a stationary point of the oscillation. The time evolution of $\left\langle\rho^{2}\right\rangle_{n, \ell}$ can therefore be obtained by substituting (16) and (17) into (12).

In the special case when $\rho_{0}=\rho_{B}$ the Laguerre-Gaussian wave functions in (15) become the Landau energy eigenstates and we obtain the constant values of $\left\langle L_{z}^{\mathrm{kin}}\right\rangle_{n, \ell}$ expected in this case [7]. In general, however, the angular momentum will oscillate around the classical value, as illustrated in Fig. 1(a) for different ratios $\rho_{0} / \rho_{B}$ and in Fig. 1(b) for different quantum numbers $\ell$ corresponding to canonical angular momenta $\ell \hbar$. If the canonical angular momentum is in the opposite direction to the magnetic field then the kinetic angular momentum may even change direction, as clearly seen for $\ell=-4$ in Fig. 1(b). We note that the moment of inertia increases with the radial quantum number $n$, resulting in larger amplitude oscillations, and that a reversal of the direction of the magnetic field corresponds to a shift of the phase of the oscillations by $180^{\circ}$.

As we have seen, the kinetic angular momentum may change with time despite the fact that the system is entirely rotationally symmetric - seemingly contradicting Noether's theorem. In order to restore angular momentum conservation we have to include the angular momentum contained in the field.
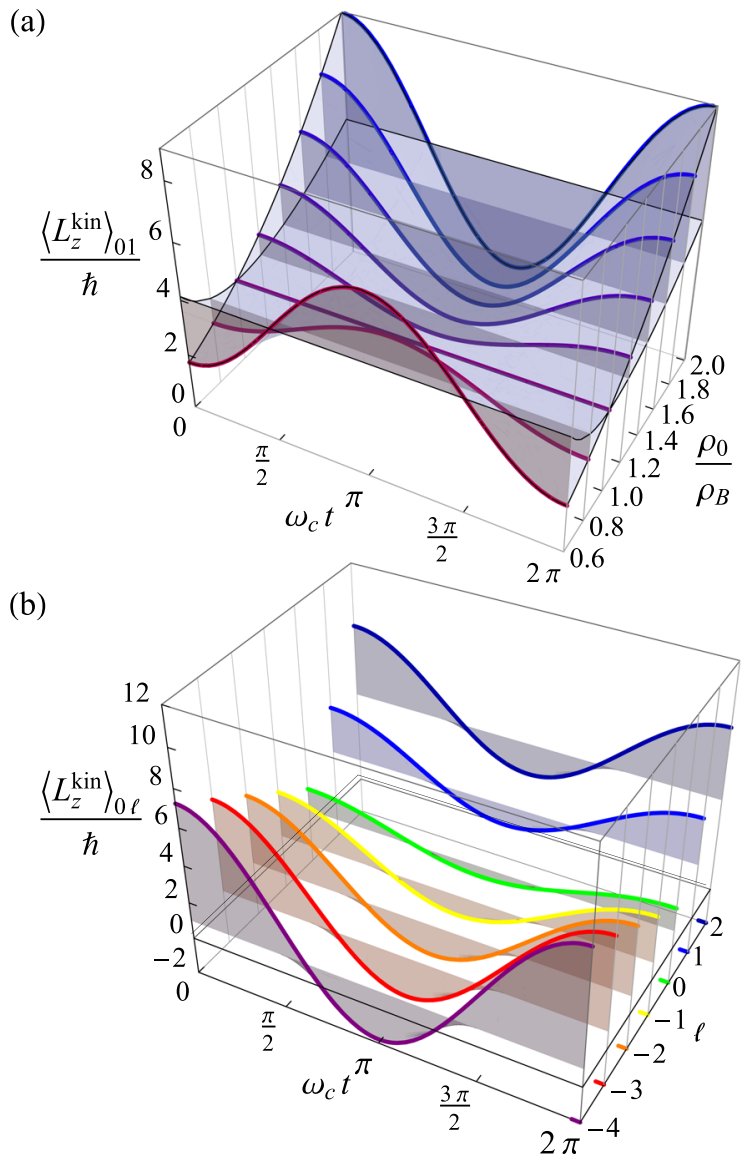

FIG. 1 (color online). Time evolution of the expectation value of the electron's kinetic orbital angular momentum for Laguerre-Gaussian states with $n=0$ and a magnetic field in the positive $z$ direction $(B>0)$. This is shown in (a) as a function of the initial width $\rho_{0}$ for $\ell=1$, and in (b) for different values of $\ell$ assuming $\rho_{0}=1.5 \rho_{B}$.

The combination of the externally imposed magnetic field and the electric field of the electron itself gives rise to a nonzero angular momentum density $\varepsilon_{0} \mathbf{r} \times(\mathbf{E} \times \mathbf{B})$ [11]. The $z$ component of the field angular momentum is

$$
\begin{aligned}
L_{z}^{\mathrm{kin} \text { field }} & =\int d V \varepsilon_{0}[\mathbf{r} \times(\mathbf{E} \times \mathbf{B})]_{z} \\
& =\int d V \varepsilon_{0}(\nabla \cdot \mathbf{E})\left(\mathbf{r} \times \mathbf{A}^{\perp}\right)_{z}
\end{aligned}
$$

[21], where $\mathbf{A}^{\perp}$ is the (manifestly gauge invariant) transverse part of the vector potential, defined by $\nabla \cdot \mathbf{A}^{\perp}=0$. In rewriting the second line we have used the fact that the electric field of the electron is longitudinal, that is, $\nabla \times \mathbf{E}=\mathbf{0}$. To complete our resolution we need only note that the first Maxwell equation is

$$
\nabla \cdot \mathbf{E}=\frac{\varrho}{\varepsilon_{0}}=\frac{e}{\varepsilon_{0}}|\Psi|^{2},
$$


where $Q$ is the charge density, so that

$$
L_{z}^{\text {kin field }}=\frac{e B}{2}\left\langle\rho^{2}\right\rangle .
$$

Using (8) and (21) the total kinetic angular momentum of the electron plus the field is, therefore,

$$
L_{z}^{\text {kin total }}=\ell \hbar=L_{z}^{\text {can }},
$$

which is conserved, as it should be. Adding the kinetic angular momentum of the field to that of the electron has restored the unique and conserved total angular momentum.

Effect of gauge transformations.-We have worked throughout in the Coulomb gauge and uncovered the result that the canonical angular momentum of the electron is equal to the total angular momentum (with the value $\ell \hbar$ ). We can and should ask what we would have found had we worked in a different gauge. The kinetic angular momentum of the field is, as we have seen, gauge invariant. To underline this point, we note that it depends only on the magnetic field $B$ and the mean-square radius $\left\langle\rho^{2}\right\rangle$, both of which are gauge invariant. Making a gauge transformation does change, however, both the vector potential and the phase of the wave function. The natural way to introduce a gauge transformation in quantum theory is through a local change in the phase of the wave function:

$$
\Psi \rightarrow e^{i \chi(\mathbf{r}, t)} \Psi
$$

In this case, the action of our canonical momentum operator on the state changes to

$$
\mathbf{p}^{\mathrm{can}} \Psi \rightarrow-i \hbar \nabla e^{i \chi(\mathbf{r}, t)} \Psi=e^{i \chi(\mathbf{r}, t)}(-i \hbar \nabla+\hbar \nabla \chi) \Psi .
$$

This change is counterbalanced by the corresponding transformation of the vector potential

$$
\mathbf{A} \rightarrow \mathbf{A}+\frac{\hbar}{e} \nabla \chi
$$

so that the kinetic momentum $\mathbf{p}^{\mathrm{kin}}$ is unchanged. The $z$ component of the canonical angular momentum is similarly changed by a gauge transformation to

$$
L_{z}^{\mathrm{can}} \rightarrow-i \hbar \frac{\partial}{\partial \phi}+\hbar \frac{\partial \chi}{\partial \phi} .
$$

Interestingly, the expectation value of this quantity for our cylindrically symmetric state will still be $\ell \hbar$, as $\left|e^{i \chi(\mathbf{r}, t)} \Psi\right|^{2}=|\Psi|^{2}$ is independent of $\phi$, and, in particular, $\langle\hbar \partial \chi / \partial \phi\rangle=0$.

Discussion.-The magnitude of the diamagnetic contribution to the kinetic angular momentum is strongly dependent on the length scale. As can be seen from (8), it is characterized by the constant $-e / 2=7.60 \times$ $10^{-4} \hbar \mathrm{T}^{-1} \mathrm{~nm}^{-2}$. In an atomic bound state with rms radius $1 \AA$, even in a field of strength $1 \mathrm{~T}$ the diamagnetic angular momentum is negligible compared to a single unit of canonical angular momentum. However, for unbound electrons, which can be distributed over a much larger area, the diamagnetic angular momentum can become significant, and may be the dominant contribution, both to the electron's kinetic angular momentum and to $E_{\perp}$. This can certainly be the case in transmission electron microscopes, where the electron beam may have a radius $\left\langle\rho^{2}\right\rangle^{1 / 2} \sim 1 \mathrm{~nm}-100 \mu \mathrm{m}$ and a field $\sim 1 \mathrm{~T}$ is provided by the objective lens. Note that in an electron microscope the radial dynamics occur in a reference frame moving with the electron along the $z$ axis [2], and so can be observed as a function of the propagation distance $[14,16]$.

While the creation of electron vortices has aroused a considerable interest in the orbital angular momentum of electron beams [22-24], little attention has been given previously to the angular momentum which arises in a magnetic field. Our diamagnetic angular momentum will occur with any electron beam, even those with no canonical orbital angular momentum. The canonical angular momentum is a manifestation of the cylindrical symmetry and is restricted to integer multiples of $\hbar$. In contrast, the diamagnetic contribution, and hence the kinetic angular momentum of the electron, may take any value.

It is interesting to ask why our electron carries two distinct angular momenta and where each of these might be expected to appear in experiment. The total angular momentum contains a part $L_{z}^{\mathrm{kin}}$ that may be ascribed to the electron, and a second part $L_{z}^{\text {kinfield }}$ that depends on both the externally imposed magnetic field and the electric field due to the electron. This latter part may be assigned either to the field, which gives the kinetic momentum, or to the electron, giving the canonical momentum. This situation is reminiscent of the linear momentum of a photon in a dielectric medium, where two rival momenta, due to Abraham and Minkowski, are the kinetic and canonical momenta [25]. As with the photon, we can associate the canonical and kinetic momenta of the electron with wavelike and particlelike properties, respectively. Hence, an electron interference pattern should reveal the $e^{i \ell \phi}$ dependence associated with the canonical angular momentum [26]. Absorption of an electron by an initially neutral target, however, should transfer the kinetic angular momentum of the electron to the rotational motion of the target, with the remaining angular momentum retained by the electric field of the now charged target.

C. G. is supported by a SUPA Prize Studentship. We also acknowledge support from the UK EPSRC under Program Grants No. EP/I012451/1 and No. EP/L002922/1, and Bridging the Gap. 
*Stephen.Barnett@glasgow.ac.uk

[1] E. Noether, Nachrichten der Königlichen Gesellschaft der Wissenschaften zu Göttingen, Math.-phys. Klasse 2, 235 (1918); D. E. Neuenschwander, Emmy Noether's Wonderful Theorem (Johns Hopkins, Baltimore, MD, 2011).

[2] K. Y. Bliokh, Y. P. Bliokh, S. Savel'ev, and F. Nori, Phys. Rev. Lett. 99, 190404 (2007).

[3] M. Uchida and A. Tonomura, Nature (London) 464, 737 (2010).

[4] J. Verbeeck, H. Tian, and P. Schattschneider, Nature (London) 467, 301 (2010).

[5] B. J. McMorran, A. Agrawal, I. M. Anderson, A. A. Herzing, H. J. Lezec, J. J. McClelland, and J. Unguris, Science 331, 192 (2011).

[6] C. Li and Q. Wang, Physica (Amsterdam) 269B, 22 (1999).

[7] K. Y. Bliokh, P. Schattschneider, J. Verbeeck, and F. Nori, Phys. Rev. X 2, 041011 (2012).

[8] P. W. Hawkes and E. Kasper, Principles of Electron Optics (Academic Press, San Diego, 1996), Vol. 1, p. 24.

[9] E. J. Konopinski, Am. J. Phys. 46, 499 (1978).

[10] J. M. Aguirregabiria and A. Hernandez, Eur. J. Phys. 2, 168 (1981).

[11] H. J. Lipkin and M. Peshkin, Phys. Lett. 118B, 385 (1982).

[12] S. M. Lloyd, M. Babiker, and J. Yuan, Phys. Rev. A 88, 031802 (2013).

[13] P. Schattschneider, Th. Schachinger, M. Stöger-Pollach, S. Löffler, A. Steiger-Thirsfeld, K. Y. Bliokh, and F. Nori, Nat. Commun. 5, 4586 (2014).
[14] G. Guzzinati, P. Schattschneider, K. Y. Bliokh, F. Nori, and J. Verbeeck, Phys. Rev. Lett. 110, 093601 (2013).

[15] G. M. Gallatin and B. McMorran, Phys. Rev. A 86, 012701 (2012).

[16] C. Greenshields, R. L. Stamps, and S. Franke-Arnold, New J. Phys. 14, 103040 (2012).

[17] I. Bialynicki-Birula, Z. Bialynicka-Birula, and C. Śliwa, Phys. Rev. A 61, 032110 (2000).

[18] L. Brillouin, Phys. Rev. 67, 260 (1945); S. P. Heims and E. T. Jaynes, Rev. Mod. Phys. 34, 143 (1962).

[19] C. Kittel, Introduction to Solid State Physics (Wiley, Hoboken, NJ, 2004).

[20] R. L. Phillips and L. C. Andrews, Appl. Opt. 22, 643 (1983).

[21] C. Cohen-Tannoudji, J. Dupont-Roc, and G. Grynberg, Photons and Atoms: Introduction to Quantum Electrodynamics (Wiley, New York, 1997), p. 45.

[22] S. M. Lloyd, M. Babiker, and J. Yuan, Phys. Rev. Lett. 108, 074802 (2012).

[23] I. P. Ivanov and D. V. Karlovets, Phys. Rev. Lett. 110, 264801 (2013).

[24] J. Verbeeck, G. Guzzinati, L. Clark, R. Juchtmans, R. Van Boxem, H. Tian., A. Béché, A. Lubk, and G. Van Tendeloo, C.R. Phys. 15, 190 (2014).

[25] S. M. Barnett, Phys. Rev. Lett. 104, 070401 (2010); S. M. Barnett and R. Loudon, Phil. Trans. R. Soc. A 368, 927 (2010).

[26] G. Guzzinati, L. Clark, A. Béché, and J. Verbeeck, Phys. Rev. A 89, 025803 (2014). 